\title{
The application of ANSYS software package in limit load analysis of structures made from anisotropic nonlinear elastic materials.
}

\author{
Nina Blokhina, ${ }^{1, *}$, and Andrey Galkin ${ }^{2}$ \\ ${ }^{1}$ Moscow state university of civil engineering, Yaroslavskoye shosse, 26, Moscow, Russia, 129337 \\ ${ }^{2}$ Acronis International GmbH, 127566, Moscow, Russia, Altufievskoye Shosse 44.
}

\begin{abstract}
The article deals with the analysis of structures made from anisotropic nonlinear elastic materials. A variation of the deformation theory of plasticity by G.A. Geniev was chosen as theoretical basis for the analysis. The article introduces the method of finding the limit load of a structure made of an anisotropic nonlinear elastic material in the state of plane stress. The analysis was carried out with the help of ANSYS software package. A special subroutine UserMat developed by the authors enables the user to specify a model in accordance with the basic theory of material behavior.
\end{abstract}

\section{Introduction}

In last two decades rapid software development has made it possible to generate computer models of quite complex processes. In structural engineering the application field of theoretical research on structural performance of materials carried out by Russian scientists towards the end of the 20th century is being expanded by new technologies.

Most modern software packages use numerical methods for structural modeling and analysis, and one of the most popular of them is the finite-element method that enables the user to create models of any complexity level. The computing power of an average PC can now suffice for the analysis of structures consisting of thousands of elements. Nevertheless, structural analysis has mostly been carried out with the use of simplified physical models. As a result, anisotropy and nonlinear properties of materials were rarely taken into account, and there was a strong need for integrate models adjusting to a larger variety of material properties.

Nowadays structural analysis is mostly performed using predetermined load analysis. Nevertheless, ultimate load analysis backed up by a certain strength theory is also an important tool in construction process. The problem is getting more complicated if physical nonlinearity and material anisotropy also have to be taken into consideration. In this way the problem can only be solved with the help of a powerful software solution with a modern

*Corresponding author: nsb_sapr@mail.ru 
user interface [1]. This article gives an example of a limit load analysis of an orthotropic flat plate performed with the help of ANSYS software package.

\section{Basic physical formulas used in the research}

The analysis is based on a variation of deformation theory of plasticity by G.A. Geniev for an orthotropic nonlinear elastic material with the same compressive strength limit parallel and across the grain for the general and special case of plane stress. [1]. According to Geniev's theory, the relations between stress and deformation are as follows:

$$
\begin{gathered}
\sigma_{x}=\frac{E_{0} \Psi}{1-v^{2}}\left(\varepsilon_{x}+v \varepsilon_{y}\right) \\
\tau_{x y}=G_{0} \Psi \gamma_{x y}, \\
\text { where } \Psi=1-\frac{\Gamma}{2 \Gamma_{s}}
\end{gathered}
$$

In this expression $\Gamma \mathrm{s}$ is the ultimate shift deformation intensity defined by the following formula:

$$
\Gamma_{s}=\Gamma_{c} \theta \sqrt{1-\sin \omega \cos \omega}
$$

where $\Gamma \mathrm{c}$ is the ultimate shift deformation intensity in uniaxial compression;

$\theta$ - the value of the stated position vector of the material resistance curve in biaxial state of stress. It can be calculated through the yield criterion of an orthotropic nonlinear elastic material shown as follows:

$$
\begin{gathered}
\frac{1}{\theta}=\sqrt{\frac{1}{\mu^{2}}+\frac{1}{\eta}-\frac{1}{\mu}} \\
\text { where } \frac{1}{\mu}=\frac{1}{2}\left[\left(\frac{1}{k_{12}}-1\right) \sin \omega+\frac{1}{k_{21}} \cos \omega\right] ; \\
\frac{1}{\eta}=\left[\frac{\sin ^{2} \omega}{k_{12}}-\sin \omega \cos \omega+\frac{\cos ^{2} \omega}{k_{21}}\right] ; \\
\frac{\tan \omega=\frac{\sigma_{2}}{\sigma_{1}}}{k_{12}}=\frac{\sin ^{2} \beta}{\rho_{1}}+\frac{\cos ^{2} \beta}{\rho_{2}} \\
\frac{1}{k_{21}}=\frac{\sin ^{2} \beta}{\rho_{2}}+\frac{\cos ^{2} \beta}{\rho_{1}}
\end{gathered}
$$




$$
\frac{1}{\rho_{1}}=\frac{R_{c}}{R_{1 s}} ; \frac{1}{\rho_{2}}=\frac{R_{c}}{R_{2 s}}
$$

$\sigma_{1}$ и $\sigma_{2}$ - main stress vectors, $\beta$ - angle between stress vector $\sigma_{1}$ and grain direction; $R_{1 t}$ - ultimate strength of the material in uniaxial tension parallel to the grain; $R_{2 t}$ - in uniaxial tension across the grain; $R_{c}$ - in compression. Methods of construction analysis based on aforementioned relations are shown in articles [2,3].

According to relation (1), $\Psi$ equals 0,5 when the structure reaches its limit state. The strength criterion (1) was developed for an orthotropic nonlinear-elastic material of uniform ultimate compressive strength, such as laminated plastic or asbestos cement. The articles describe the analysis of an asbestos cement flat plate under plane stress. The plate of dimensions $2000 \times 2000 \mathrm{~mm}$ is fixed at the bottom edge and has a square orifice $400 \times 400$ $\mathrm{mm}$. The central part of the plate, $1 / 4$ of its surface, is subject to a uniformly distributed vertical load. Physical constants required in the analysis are as follows: $R_{l t}=15 \mathrm{MPa}, R_{2 t}$ $=11 \mathrm{MPa}, R_{c}=46 \mathrm{MPa}$. The Poisson coefficient equals 0,2 ; initial elastic modulus $E_{0}=$ $20000 \mathrm{MPa}$. In order to find the limit load in accordance with the strength criterion of an orthotropic nonlinear-elastic material we need to introduce a relation of minimal $\Psi$ of the whole structure to the load applied to the structure. The relation can be displayed in a form of function

$$
\Psi_{\text {min }}=f(P)
$$

where P coefficient is used for varying the load. The limit load problem is solved by finding the value of $\mathrm{P}$ (with precision rate $\varepsilon$ ) with which $\Psi$ min is maximally close to the limit value $(0,5)$ for the element given. Numerical solutions have proved that function (3) is monotonically decreasing.

\section{Computational analysis methods}

The article [4] gives an example of applying the aforementioned theory to structural analysis with the help of ANSYS. The analysis is carried out via UserMat, a user subroutine which enables the user to specify a certain material. Thus an implementation model of Geniev's theory for a specific material is created. ANSYS also provides built-in interface for calculating 'userfc', user-defined failure criterion. Nevertheless it has a number of disadvantages such as the absence of parallel computing applications or doubling the number of computations required. So the authors have decided to develop a software solution for automating limit load analysis which has no disadvantages of this kind. First of all UserMat subroutine was modified so that the obtained $\Psi$ can be exported into a file. The easiest way to support parallel computation was to export $\Psi$ into numerous files at each iteration in order to avoid different process flows competing for files.

The software solution developed by the authors interacts with ANSYS in the following way:

- Adjusts the macro for ANSYS by changing P coefficient with the help of SetNewCoef subroutine; 
- Initiates the ANSYS analysis using ExecuteAnalysis subroutine;

- Reads the generated files that contain $\Psi$ and finds its minimal value $\Psi$ min using ReadMinPsi.

The basic element of the solution is TryFindFsi, a recursive subroutine that initiates computation cycle for calculating $\Psi$ value. If $\Psi$ turns out to be maximally close to 0,5 , the subroutine shuts down. If not, it reduces increment and calls itself again from the current point. An important part of the analysis is the comparison of linear and nonlinear analysis results. Fig. 1 shows contour plots of vertical stresses for a linear elastic solution. Fig.2 shows the results of nonlinear analysis. In case of nonlinear solution peak stresses in their concentration areas are being smoothed out. Further comparison also demonstrates that peak stress tend to smooth out more as the load approaches the limit value.

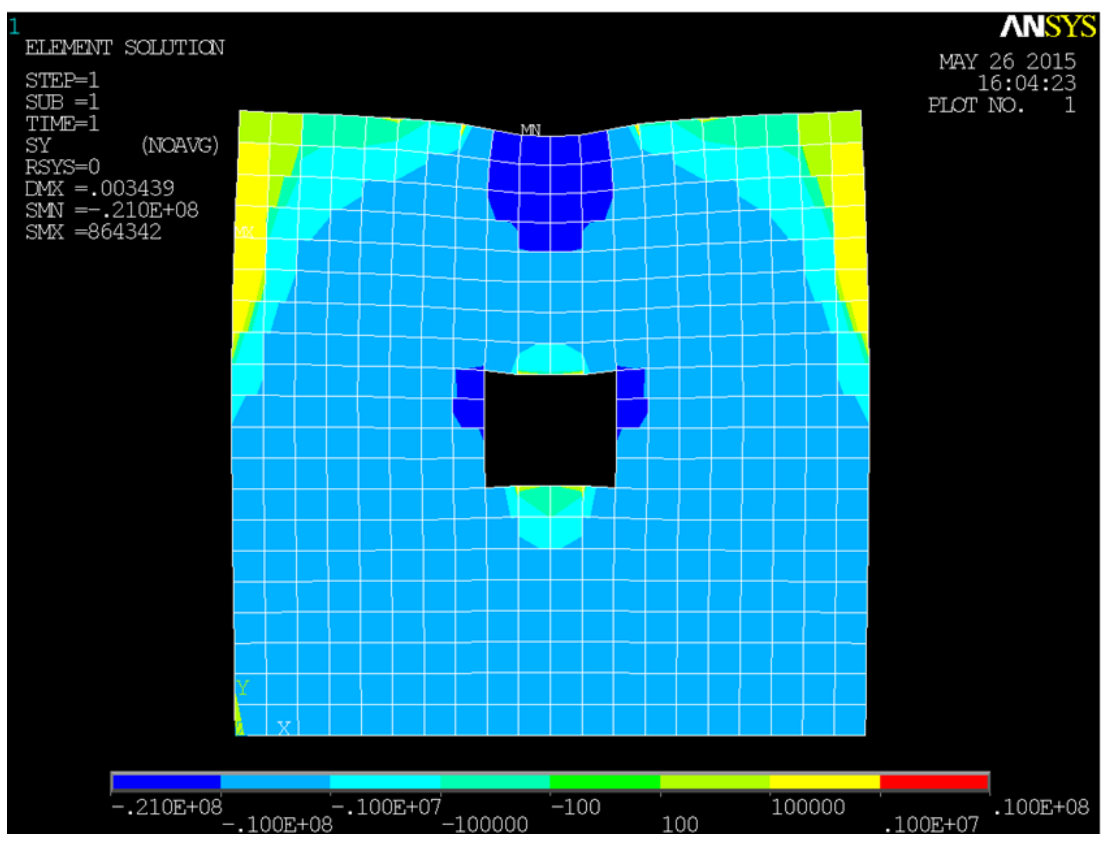

Fig.1 Contour plots of vertical stresses in linear elasic solution 


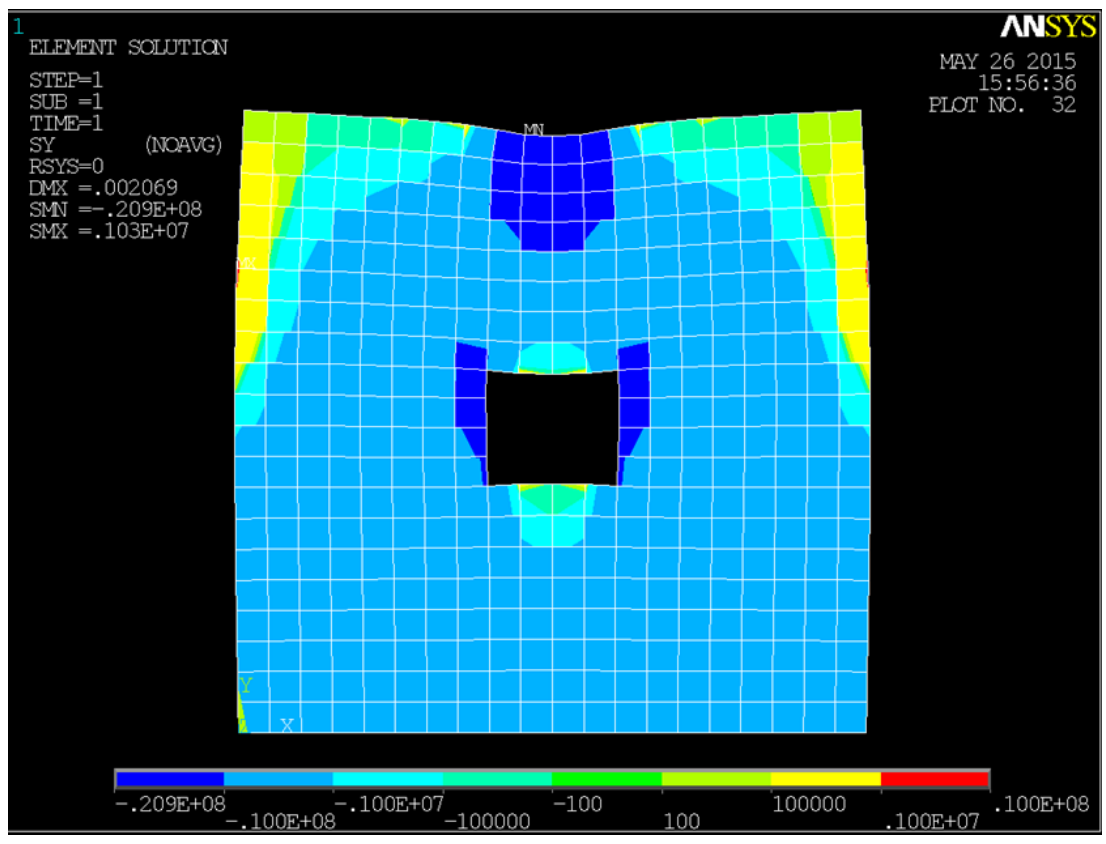

Fig.2 Contour plots of vertical stresses in nonlinear solution

\section{References}

1. G.A.Geniev, A.S. Kurbatov, F.A. Samedov. Voprosy prochnosti I plastichnosti anizotropnykh materialov [Anisotropic Materials Strength and Plasticity]. Moscow. Interbook Publ., (1993)

2. N.S. Blokhina, Applied Mechanics and Materials, 405-408, 2686 (2013)

3. N.S Blokhina, ASV Publ. 6, 384 (2011)

4. N.S. Blokhina, A. G. Galkin, S.V. Scherbina, Structural Mechanics and Analysis of Constructions, 2, 3 (2015) 